\title{
CURVATURE AND THE BACKWARD SHIFT OPERATORS
}

\author{
G. MISRA
}

\begin{abstract}
Let $\varphi_{\alpha}$ be a Möbius transformation of the unit disk $\mathbf{D},|\alpha|<1$. We characterize all the operators $T$ in $B_{1}(\mathbf{D})$ which are unitarily equivalent to $\varphi_{\alpha}(T)$ for all $\alpha$ with $|\alpha|<1$, using curvature techniques.
\end{abstract}

0. Introduction. The backward shift operator $U_{+}^{*}$ lies in the class $B_{1}(\mathbf{D})$, first introduced in Cowen and Douglas [1]. It is easy to compute the curvature $K_{U_{+}^{*}}(\omega)$, which turns out to be $-\left(1-|\omega|^{2}\right)^{-2}$. For any operator $T$ in $B_{1}(\mathbf{D})$ with $\|T\| \leq 1$, we have $[\mathbf{3}], K_{T}(\omega) \leq-\left(1-|\omega|^{2}\right)^{-2}$. This inequality is best possible over all of $\mathbf{D}$ since equality holds for $T=U_{+}^{*}$. Some time back R. G. Douglas asked if the inequality is best possible pointwise; that is, if $T \in B_{1}(\mathbf{D}),\|T\| \leq 1$ and $\dot{K}_{T}\left(\omega_{0}\right)=-\left(1-\left|\omega_{0}\right|^{2}\right)^{-2}$ for some $\omega_{0}$ in $\mathbf{D}$, does it follow that $T$ is unitarily equivalent to $U_{+}^{*}$ ?

In this note we obtain a characterization of those operators $T$ in $B_{1}(\mathbf{D})$ that are unitarily equivalent to $\varphi_{\alpha}(T)$ for all $\alpha$, where $\varphi_{\alpha}$ is a Möbius transformation of the disk, and answer the above problem in the negative.

1. The class $B_{1}(\mathbf{D})$ is defined as follows.

$$
\begin{aligned}
& B_{1}(\mathbf{D})=\{T \in \mathcal{L}(\mathcal{H}): \text { (i) } \mathbf{D} \subset \sigma(T), \\
& \text { (ii) } \bigvee_{\omega \in \mathbf{D}} \operatorname{ker}(T-\omega)=\mathcal{H}, \\
& \text { (iii) } \operatorname{ran}(T-\omega)=\mathcal{H}, \\
&\text { (iv) } \operatorname{dim} \operatorname{ker}(T-\omega)=1 \text { for all } \omega \in \mathbf{D}\} .
\end{aligned}
$$

For each operator $T$ in $B_{1}(\mathbf{D})$, such that $T(\gamma(\omega))=\omega \gamma(\omega)$, it is possible to find a holomorphic family of eigenvectors $\gamma(\omega)$ on D. Following Cowen and Douglas [1], we can define the curvature of an operator $T$ in $B_{1}(\mathbf{D})$ to be

$$
K_{T}(\omega)=\frac{\partial^{2}}{\partial \omega \partial \bar{\omega}} \log \|\gamma(\omega)\|^{-2}
$$

Let $\varphi_{\alpha}(\omega)=(\alpha-\omega)(1-\bar{\alpha} \omega)^{-1}$ be a Möbius transformation of the unit disk, $|\alpha|<1$. Whenever $\|T\| \leq 1$, the operator $\varphi_{\alpha}(T)$ is well defined and a simple application of chain rule yields

$$
\|\left.\varphi_{\alpha}^{\prime}(\omega)\right|^{2} K_{\varphi_{\alpha}(T)}\left(\varphi_{\alpha}(\omega)\right)=K_{T}(\omega) .
$$

In particular if $T=U_{+}^{*}$, we obtain

$$
\begin{aligned}
K_{\varphi_{\alpha}\left(U_{+}^{*}\right)}\left(\varphi_{\alpha}(\omega)\right) & =\left|\varphi_{\alpha}^{\prime}(\omega)\right|^{-2} K_{U_{+}^{*}}(\omega)=-\left|\varphi_{\alpha}^{\prime}(\omega)\right|^{-2}\left(1-|\omega|^{2}\right)^{-2} \\
& =-\left(1-\left|\varphi_{\alpha}(\omega)\right|^{2}\right)^{-2}=K_{U_{+}^{*}}\left(\varphi_{\alpha}(\omega)\right) .
\end{aligned}
$$

Received by the editors June 28, 1983.

1980 Mathematics Subject Classification. Primary 47B37. 
The Cowen-Douglas theorem, which states that two operators in $B_{1}(\mathbf{D})$ are unitarily equivalent if and only if their curvatures are equal, implies $\varphi_{\alpha}\left(U_{+}^{*}\right)$ is unitarily equivalent to $U_{+}^{*}$ for all $\alpha$. We can now ask ourselves, which other operators in $B_{1}(\mathbf{D})$ share this property.

Proposition. If $T$ is in $B_{1}(\mathbf{D})$ and $\|T\| \leq 1$ then $\varphi_{\alpha}(T)$ is unitarily equivalent to $T$ for all $\alpha$ if and only if

$$
\mathcal{K}_{T}(\omega)=-c\left(1-|\omega|^{2}\right)^{-2}
$$

for some constant $c \geq 1$.

PROOF. if $K_{T}(\omega)=-c\left(1-|\omega|^{2}\right)^{-2}$, a calculation similar to the one above shows that $\varphi_{\alpha}(T)$ must be unitarily equivalent to $T$ for all $\alpha$.

Conversely, if $T$ is unitarily equivalent to $\varphi_{\alpha}(T)$ for all $\alpha$ then we must have

$$
K_{\varphi_{\alpha}(T)}\left(\varphi_{\alpha}(\omega)\right)=\left|\varphi_{\alpha}^{\prime}(\omega)\right|^{2} \mathcal{K}_{T}(\omega)=\mathcal{K}_{T}\left(\varphi_{\alpha}(\omega)\right) .
$$

In particular, $\left|\varphi_{\alpha}^{\prime}(0)\right|^{-2} K_{T}(0)=K_{T}(\alpha)$ so $K_{T}(\alpha)=\left(|\alpha|^{2}-1\right)^{-2} K_{T}(0)$ for all $\alpha$ in D. Let $c$ equal $K_{T}(0)$, then $K_{T}(\omega) \leq-\left(1-|\omega|^{2}\right)^{-2}$ implies that $c \geq 1$.

Now, consider the weighted shift operator $T$ with weights $\omega_{n}=\left(c_{n} / c_{n+1}\right)^{1 / 2}$, where $c_{n}$ is the $n$th coefficient in the generalized binomial expansion of $\left(1-|\omega|^{2}\right)^{-c}$ for a fixed real number $c$. The adjoint of $T$ is in $B_{1}(\mathbf{D})$ (Seddighi $[\mathbf{4}]$ ) and $\gamma(\omega)=$ $\left(1-|\omega|^{2}\right)^{-c}$ is a holomorphic family of eigenvectors for $T^{*}$. It is easy to compute

$$
\mathcal{K}_{T^{*}}(\omega)=-c\left(1-|\omega|^{2}\right)^{-2} .
$$

When $c$ is an even integer these operators can be identified with the adjoint of multiplication on the Hilbert space of square integrable holomorphic functions on D with respect to the measure $d \mu=(i / 2)\left(1-|\omega|^{2}\right)^{2-2 q} d \omega \wedge d \bar{\omega}$ (cf. Kra [2, pp. 89 and 95]). Thus, we are able to idenify all of the operators that are unitarily equivalent to all their Möbius transforms $\varphi_{\alpha}(T)$.

It follows from the Proposition that if $T \in B_{1}(\mathbf{D})$ and $\|T\| \leq 1$, then the following two statements are equivalent.

(1) $\mathcal{K}_{T}\left(\omega_{0}\right)=-\left(1-\left|\omega_{0}\right|^{2}\right)^{-2}$ for some $\omega_{0}$ and $\varphi_{\alpha}(T)$ is unitarily equivalent to $T$ for all $\alpha$.

(2) $T$ is unitarily equivalent to $U_{+}^{*}$.

However, $\mathcal{K}_{T}\left(\omega_{0}\right)=-\left(1-\left|\omega_{0}\right|^{2}\right)^{-2}$ does not necessarily imply that $T$ is unitarily equivalent to $U_{+}^{*}$ as we will show by means of an example.

Let $T$ be a weighted shift operator with weights $\omega_{0}, \omega_{1}, \omega_{2}, \ldots$ We can consider $T$ to be an ordinary shift on a weighted sequence space (Shields [5]) with weights $\beta(0), \beta(1), \ldots$ For $\omega \in \mathbf{D}$,

$$
\gamma(\omega)=\left(\frac{1}{\beta(0)}, \frac{\omega}{\beta(1)}, \frac{\omega^{2}}{\beta(2)}, \ldots\right)
$$

is an eigenvector for $T^{*}$ and

$$
\|\gamma(\omega)\|^{2}=\sum_{n=0}^{\infty} \frac{|\omega|^{2 n}}{|\beta(n)|^{2}} .
$$


Assuming $T^{*}$ is in $B_{1}(\mathbf{D})$ (Seddighi [4] determines when a weighted shift is in $\left.B_{1}(\mathbf{D})\right)$, we compute

$$
\begin{aligned}
\mathcal{K}_{T^{*}}(\omega)=- & {\left[\left(\sum_{n=0}^{\infty}(n+1)^{2} \frac{|\omega|^{2 n}}{|\beta(n+1)|^{2}}\right)\left(\sum_{n=0}^{\infty} \frac{|\omega|^{2 n}}{|\beta(n)|^{2}}\right)\right.} \\
& \left.-|\omega|^{2}\left(\sum_{n=0}^{\infty}(n+1) \frac{|\omega|^{2 n}}{|\beta(n+1)|^{2}}\right)^{2}\right]\left[\sum_{n=0}^{\infty} \frac{|\omega|^{2 n}}{|\beta(n)|^{2}}\right]^{-2} .
\end{aligned}
$$

Putting $\omega=0$, we see that

$$
K_{T^{*}}(0)=-\left|\beta(0) \beta(1)^{-1}\right|^{2}
$$

Now, let $T$ be the weighted shift with weights $1, \frac{1}{2}, 1,1,1, \ldots$ It is easy to verify that $T^{*} \in B_{1}(\mathbf{D})$ and $\left\|T^{*}\right\|=1$. Since $\beta(0)=1$ and $\beta(1)=1$, it follows that $K_{T^{*}}(0)=-1$. Obviously $T^{*}$ is not unitarily equivalent to $U_{+}^{*}$.

In fact, we can compute $h_{T^{*}}(\omega)$ explicitly for the weighted shift of our example and show that $h_{T^{*}}\left(\varphi_{\alpha}(\omega)\right) \not \equiv\left|\varphi_{\alpha}^{\prime}(\omega)\right| h_{T^{*}}(\omega)$, therefore $T$ is not unitarily equivalent to $\varphi_{\alpha}(T)$ for any $\alpha$.

\section{REFERENCES}

1. M. J. Cowen and R. G. Douglas, Complex geometry and operator theory, Acta. Math. 141 (1978), 187-261.

2. I. Kra, Automorphic functions and Kleinian groups, Benjamin, New York, 1972.

3. G. Misra, Curvature inequality and extremal properties of bundle shifts, J. Operator Theory (to appear).

4. K. Seddighi, Essential spectra of operators in the class $B_{n}(\Omega)$, Proc. Amer. Math. Soc. 87 (1983), $453-458$.

5. A. L. Shields, Weighted shift operators and analytic function theory, Topics in Operator Theory (C. Pearcy, ed.), Math. Surveys, no. 13. Amer. Math. Soc.. Providence, R.I.. 1974, pp. 51-128.

Department of Mathematics. Iniversity of Georgia. Athens, Georgia 30602 